\title{
Identification of Small Molecule Memapsin Inhibitors via Computation-based Virtual Screening
}

\author{
Afaf H. Al-Nadaf ${ }^{1, *}$, Mutasem O. Taha ${ }^{2}$ \\ ${ }^{1}$ Department of Medicinal Chemistry and Pharmacognosy, Faculty of Pharmacy, Applied Science University, Jordan \\ ${ }^{2}$ Drug Discovery Unit, Department of Pharmaceutical Sciences, Faculty of Pharmacy, University of Jordan, Jordan
}

Copyright $(\mathcal{C} 2015$ by authors, all rights reserved. Authors agree that this article remains permanently open access under the terms of the Creative Commons Attribution License 4.0 International License

\begin{abstract}
Background: Alzheimer's disease (AD) is a degenerative disease of the brain common form of dementia. A variety of therapeutic strategies for modulating the progression or prevention of $\mathrm{AD}$ are currently being investigated. The etiology of the disease is characterized by aggregates of amyloid plaques, largely composed of amyloid- $\beta$ peptide formed from the amyloid precursor protein cleaved by Memapsin 2(Beta-secretase / BACE1). Based on its key role in the $\beta$-amyloid cascade, inhibition of Memapsin 2 is widely recognized as one of the most promising therapeutic approaches for the treatment and prevention of AD. However, the development of small molecule, brain penetrant Memapsin 2 inhibitors has been challenging. Method: Molecular docking study using LigandFit Docking and Scoring as well as LibDock Docking functions were performed as a preliminary in-silico screening test for National Cancer Institute (NCI) database using binding pocket of Memapsin. Followed by in-vitro enzyme inhibition assay, High-ranking docked conformers and poses were scored using seven scoring functions. The validation for our docking-scoring procedure was performed through employing the same conditions to dock a well-known Memapsin inhibitor IO2. High ranking compounds were evaluated in vitro using Memapsin fluorescence resonance energy transfer (FRET) assay. Results: The docking simulation resulted in a close model to the crystallographic structure. Five of the important interactions are shared between the co-crystallized ligand and in-silico hits. Virtual screening identified low micromolar inhibitory leads from the NCI list of compounds. The most potent hit exhibited Memapsin $\mathrm{IC}_{50}$ values of 11.1 $\mu \mathrm{M}$ in enzymatic assay. Conclusion: We have identified a low micro-molar Memapsin inhibitor with $\mathrm{IC}_{50}$ of $11.1 \mu \mathrm{M}$. Our results suggest that in silico high-throughput screening approach can serve as useful source to identify new hits which can be used as lead candidate for synthetic modification in order to reach more potent enzyme inhibitors.
\end{abstract}

Keywords Alzheimer's Disease, Memapsin 2 Inhibitors, Docking, Virtual Screening, In vitro Validation

\section{Introduction}

Alzheimer's disease (AD) currently upset more than 27 million people worldwide, and its population is predicted to grow, creating a major economic and emotional dilemma on society [1]. At present only symptomatic treatments with modest effectiveness are available. Even in the absence of a diagnosable disorder of cognition, the perception of increased forgetfulness and declining memory function is a clear source of sorrow, embarrassment, and low self respect in the elderly [2-4].

$\mathrm{AD}$, is caused by the formation of neuritic plaques in the brain and neurofibrillary tangles in the nerves. In neuritic plaques the fibrils are formed by a protein known as amyloid $\beta$-protein $(A \beta)[5-8]$. A $\beta$ is derived from proteolytic cleavage of the membrane bound amyloid precursor protein (APP). APP is produced by two ways, first: nonamyloidgenic where APP is cleaved by $\alpha$-secretase to yield soluble APP $\alpha$.The cleavage site is within the $A \beta$ sequence, thereby precluding its formation. Second: amyloidgenic where APP is cleaved by Memapsin 2 to yield soluble APP $\beta$ and also A $\beta$. Cleavage of APP by $\gamma$-secretase is common to both pathways. A serie of reports suggest that the activity of Memapsin is upregulated in late-onset $\mathrm{AD}$ brains and might be considered the primary target for lowering $A \beta .[6-8]$.

Memapsin 2 is a membrane bound aspartyl protease [9], which is amenable to structure-based drug design [10]. Though, the identification of small molecule Memapsin inhibitors that penetrate the CNS and lower CNS-derived $A \beta$ has proved to be exceptionally challenging. Memapsin 2 null mice are viable and do not produce amyloid plaques upon aging, suggesting that small-molecule inhibitors of Memapsin 2 would be potential disease modifying agents for $\mathrm{AD}$.

Based on the pathophysiology, various pharmacologic approaches are developed for the treatment of $\mathrm{AD}$. The approved treatment strategies provide symptomatic improvement in $\mathrm{AD}$. The therapies under evaluation for the 
treatment of AD have disease modifying and neuro-protective approaches [11-14]. Early interest in developing therapies for $\mathrm{AD}$ focused on the cholinergic system as disease progression is known to be accompanied by loss of cholinergic neurons $[13,15]$. In reality, cholinergic deficit and $A \beta$ levels correlate well in the disease state $[16,17]$. Memantine (an NMDA receptor antagonist) apart, all current FDA-approved therapies for the symptomatic treatment of $\mathrm{AD}$ are acetylcholine esterase inhibitors (AChEIs) [18-19]. However, most of the clinical efficacy observed in $\mathrm{AD}$ is largely restricted to the first two years from inception of treatment. Moreover, AChEIs have a number of undesirable side effects, including nausea, sweating, salivation and gastrointestinal disturbances [13,18-19]. Therefore, there remains a significant need for treatments with less side effect potential, or, preferably, treatments targeting AD progression.

Several classes of Memapsin 2 inhibitors were recently reported [20-27]. More recently, several nanomolar transition state analogues (TSA) Memapsin inhibitors were reported [25,28, 29] . Clearly, the main focus of recent efforts toward the development of new Memapsin inhibitors concentrate on structure-based ligand design [27-28]. To date, several human Memapsin X-ray complexes are documented in the Protein Data Bank [29-32].

In the present work we applied docking-based virtual screening approach to find possible low molecular weight hits that have been used before as search query for drug data base [51]. This search has been followed by scoring and binding energy calculation as pre-filtration step before in vitro assay screening. We reported the successful application of our in-silico high-throughput docking approach in the screening of about 238,819 compounds existing in National Cancer Institute (NCI) database, which has resulted in the discovery of a series of nonpeptide Memapsin inhibitors with different schaffolds this methodology succeeded before in our recent work which resulted in discovery of accepted inhibitory activity for Memapsin by haloperidol antipcychotic [51]. We hope that this work can be optimized by us or other researchers in search parameters or filtration steps as well as synthesis approach to develop new potent low molecular weight Memapsin inhibitors.

\section{Materials and Methods}

For all information about software refer to supporting data S1.1

All information about docking conditions and enzyme inhibition assay refer to supporting data (S1.2-S.2.4) which is the same procedure as reference [51] with slight modifications

\section{Results}

To treasure novel low molecular weight compounds as starting points for lead optimization is a major challenge in drug discovery. In the present work, we were interested in identifying low molecular-weight hits which usually have no known pharmacological activity, but available as synthesized compounds in the NCI library. We aim to test these compounds against Memapsin at the same time we aim to reduce false positive hits using in-silico search. This approach also aim to decrease time and chemicals used so that drug discovery will need less time and less chemical waste, as well as get benefit of so many chemical compounds already found in the universe to treat diseases.

\subsection{Molecular Modeling}

The central assumption of structure-based design is that good inhibitors must possess significant structural and chemical complementarity to their target receptor.

Docking simulations were performed with the inhibitors: IO2

(3-\{5-[(1r)-1-Amino-1-Methyl-2-Phenylethyl]-1,3,4-Oxadia zol-2-Y1\}-N-[(1r)-1-(4-Fluorophenyl)Ethyl]-5-[Methyl(Met hylsulfonyl) Amino] Benzamide) in 2IRZ complex and F2I (N'-\{(1s,2r)-1-(3,5-Difluorobenzyl)-2-Hydroxy-

3-[(3-Iodobenzyl)Amino]Propyl $\}$-5-Methyl-N,N-

Dipropylisophthalamide) present in the 2IQG complexe from PDB, in order to compare the solutions obtained in silico with the orientations of the inhibitors, bound to the Memapsin active site in the crystallographic complexes. First, these ligands were extracted from the complex and then submitted to the simulation after adding hydrogen atoms to the Memapsin enzyme structure.

In Figure (1), it is possible to observe that the orientations of highest score obtained by flexible docking for IO2 inhibitor is very similar to the crystallographic orientations. In this way, this docking method is able to reproduce experimental binding modes of molecules from NCI. Moreover inhibitor F2I was abstracted from its protein 2IQG and docked to 2IRZ using same docking conditions used in our in silico search. We believe that this will validate our finding about possible hits will act in similar way as this known inhibitor out of his own protein far a way that IO2 is already co-crystallized with 2 IRZ so that the binding site taking the shape for this particular inhibitor.

We carried out virtual screens of the National Cancer Institute (NCI) database (http://129.43.27.140/ ncidb2) for chemical compounds that could possibly bind to the detected cavity. This database comprises the coordinates of about 250,251 small chemical compounds. To eliminate the large amount of inappropriate compounds we used filtered by Lipinski's [47] and Veber's criteria [48] prefiltration step prior docking. Remaining hits were subjected to ligandfit and libdock docking experiments. High-ranking docked conformers and poses were scored using seven scoring functions: Jain [37], LigScore1, LigScore2 [35, 38], PLP1 [39], PLP2 [39], PMF, and PMF04 [40-42]. Subsequently binding energy was calculated to end up with 341 hits.

Out of the 40 highest-ranking hits based on consensus 
scoring values simultaneously with binding free energy (nominated based on availability from the NCI ; cost of screening and different consensus scoring) acquired for experimental validation 6 were found to possess inhibitory activities against Memapsin (fig. 3 ) with $\mathrm{IC}_{50}$ for most potent compound 3 about $11 \mu \mathrm{M}$.

\subsection{Effect of Hits on Memapsin Activity}

To evaluate the inhibitory effect of captured compounds against Memapsin, an in vitro Memapsin inhibitory assay was conducted. In this inhibitory assay; the concentration that inhibits $50 \%$ of the enzyme, $\mathrm{IC}_{50}$, was measured. Table 1 shows the $\mathrm{IC}_{50}$ of active compounds. The validity of the test was established by testing the inhibitory action of the standard inhibitor A1847 on Memapsin, which showed an $\mathrm{IC}_{50}$ value of $40 \mathrm{nM}$ that is comparable to the published value [49].

\section{Discussion}

The need for a rapid search for small molecules that may bind to targets of biological interest is of a vital importance in the drug discovery process. One way of achieving this is the in-silico or virtual screening of large compound collections to identify a subset of compounds that contains relatively many hits against the target, compared to a random selection from the collection. The compounds that are virtually screened can stem from corporate or commercial compound collections, or from virtual compound libraries. If a three-dimensional structure or model of the target is available, a commonly used technique is structure-based virtual screening.

To discover new inhibitors for Memapsin we started via structure-based virtual screening. A high-throughput screening study was presented against Memapsin receptor in which LigandFit combined with LibDock, of Discovery studio (Version 2.5, Accelry's Software Inc.) was applied. The software allows us to virtually screen a database of compounds and predict the strongest binders based on various scoring functions.

Docking simulations end with 6 compounds that were able to inhibit the activity of Memapsin. Validation for our technique was through, concomitant docking of IO2 and F2I (which are knowen memapsin inhibitors) in 2IRZ protein. Orientations by flexible docking for IO2 inhibitor of highest score obtained are very similar to the crystallographic orientations, Figure 1. Moreover the molecular interactions of the highest ranking binding mode of IO2 summarized in Figure 2, illustrate multiple contact points. Obviously, the (-NH2) group of $\mathbf{I O 2}$ interacts with acidic moiety of ASP228 and ASP32 via electrostatic interaction, as bi-dentate like configuration. Benzyl side chain interacts with aromatic binding sit represented by PHE108 and TRP115 and THR72 hydroxyl networks oxadiazole nitrogen via hydrogen bond interaction. Hydrogen bond interaction can be observed in other positions in particular: GLN73 with carbonyl fragment; ARG235 and THR232 with (-SO2) fragment as well as GLY230 with amidic (-NH) portion of IO2. Other type of interactions can be noticed with aromatic ring holding chlorine in IO2 and TYR14 as well as THR231 which is clearly hydrophobic interaction. At the end Vander wall bond can be seen between LEU30 and benzyl (-CH2) in IO2 .

Table 1. Active Hits Captured by ligandfit : Their Corresponding scoring values; binding energy and in Vitro Bioactivities

\begin{tabular}{|c|c|c|c|c|c|c|c|c|c|}
\hline $\begin{array}{c}\text { Compound } \\
\text { Name }^{\mathbf{d}}\end{array}$ & $\begin{array}{c}\text { Binding } \\
\text { Energy }^{\mathbf{a}} \\
(\mathrm{kcal} / \mathrm{mol})\end{array}$ & Consensus $^{\mathbf{b}}$ & PLP1 $^{\mathbf{b}}$ & PLP2 $^{\mathbf{b}}$ & Jain $^{\mathbf{b}}$ & PMF $^{\mathbf{b}}$ & LigScore1 $^{\mathbf{b}}$ & LigScore2 $^{\mathbf{b}}$ & $\begin{array}{c}\text { IC }_{50}{ }^{\mathbf{2}} \\
\left(\mathrm{micM}^{\mathbf{2}}\right.\end{array}$ \\
\hline $\mathbf{1}$ & -141.474 & 8 & 97.3 & 101.23 & 5.83 & 124.27 & 6.46 & 6.94 & $177.7 \mathbf{X}_{10}$ \\
\hline $\mathbf{2}$ & -201.93 & 8 & 112.02 & 119.44 & 7.82 & 145.53 & 7.32 & 6.67 & $104.7 \mathrm{X} 10^{3}$ \\
\hline $\mathbf{3}$ & $\underline{-224.02}$ & $\underline{7}$ & $\underline{83.62}$ & $\underline{94.17}$ & $\underline{6.82}$ & $\underline{118.68}$ & $\underline{7.27}$ & $\underline{7.27}$ & $\underline{11.17}$ \\
\hline $\mathbf{4}$ & -208.312 & 6 & 90.42 & 92.2 & 4.66 & 122.22 & 5.98 & 6.58 & 172.3 \\
\hline $\mathbf{5}$ & -134.001 & 3 & 88.36 & 88.58 & 6.89 & 127.1 & 3.9 & 4.41 & $282.3 \times 10^{2}$ \\
\hline $\mathbf{6}$ & -158.059 & 1 & 85.12 & 73.46 & 3.44 & 80.03 & 5.01 & 5.32 & $108.9 \times 10^{2}$ \\
\hline $\mathbf{F 2 I}$ & -170.875 & 6 & 124.06 & 123.51 & 10.34 & 145.59 & 6.31 & 5.29 & - \\
\hline
\end{tabular}

a: binding Energy calculated by catalyst software.

b: seven scoring functions implemented in ligandfit / Cerius(2).

c: $\mathrm{IC}_{50}$ calculated by in-vitro enzyme assay

d: compounds structure in figure 3 


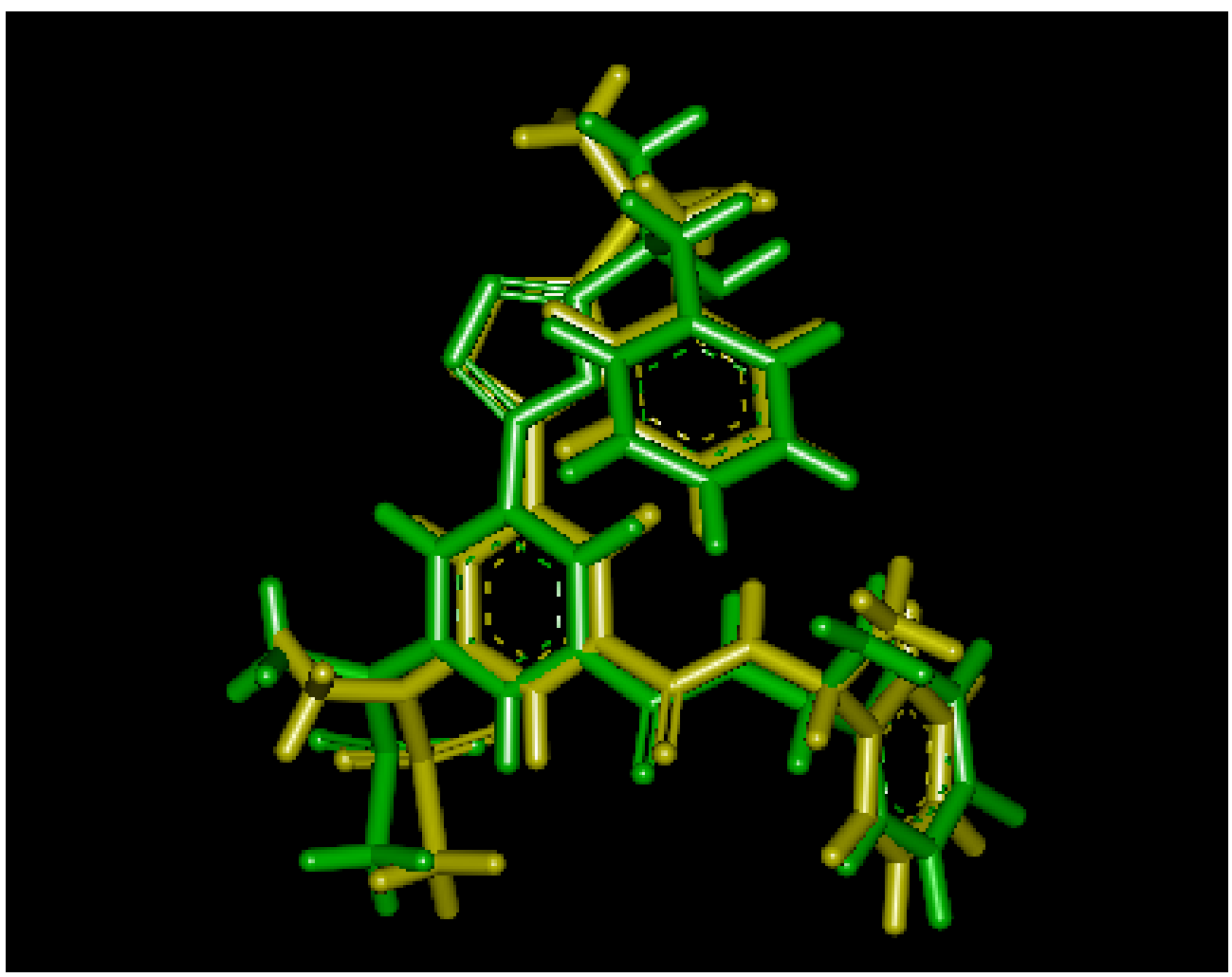

Figure 1. Comparison between the docked conformer/pose of inhibitor IO2 (IC50=12 nM) (Green) as produced by the docking simulation and the crystallographic structure (Yellow) of this inhibitor within Memapsin ( PDB code: 2IRZ)

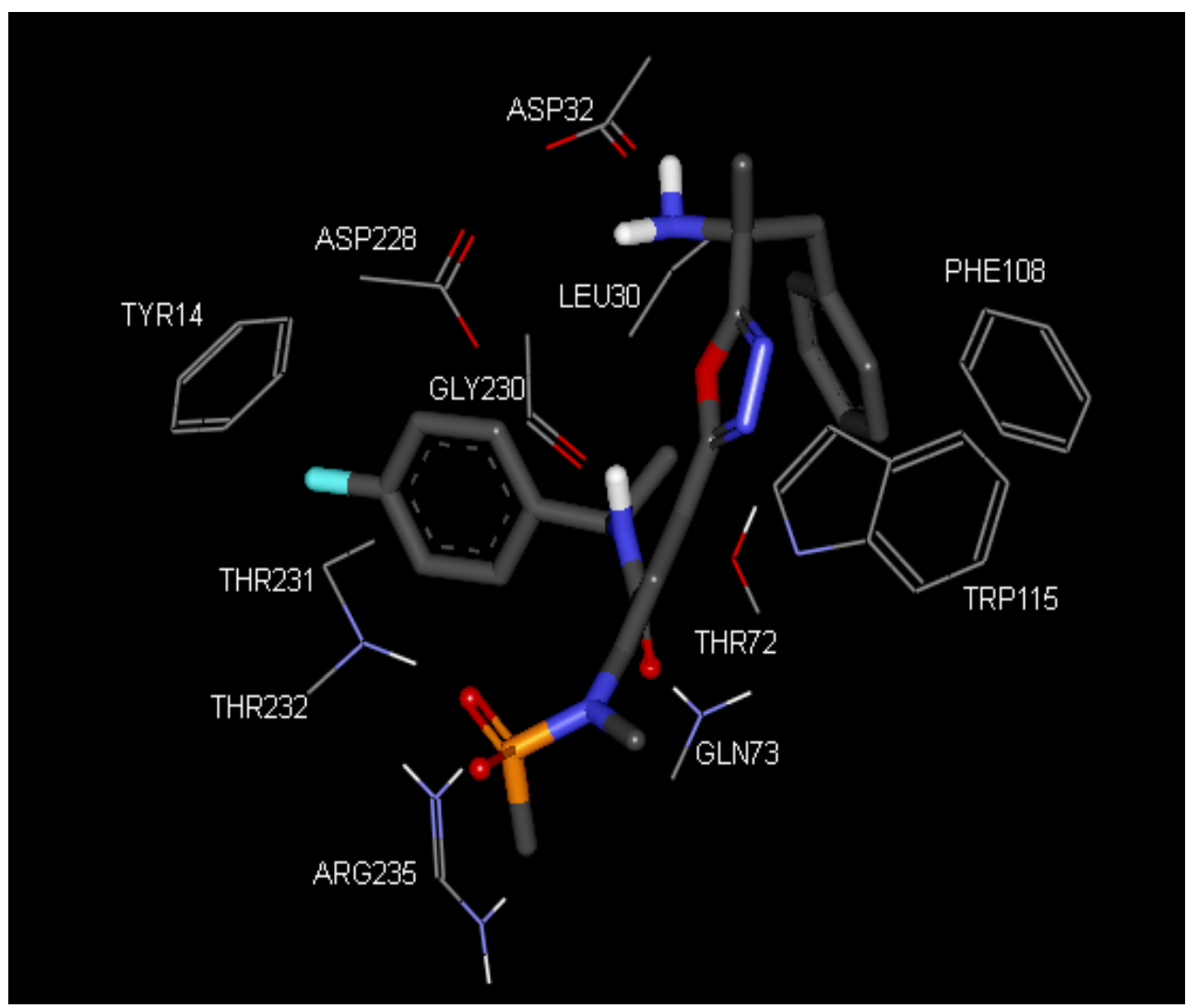

Figure 2. (A) Detailed view of the co-crystallized structure $\mathbf{I O 2}\left(\mathrm{IC}_{\mathbf{5 0}}=12 \mathrm{nM}\right)$ and the corresponding interacting amino acids within the binding site of Memapsin (PDB code: 2IRZ) . 
<smiles>Cc1ccc(NCCNCC(C)(C)O)c2c(=O)c3ccc(Cl)cc3sc12</smiles>

CI0038113

1<smiles>CC1OC(OC2CCc3c(O)c4c(c(O)c3C2)C(=O)c2ccccc2C4=O)CC(N)C1O</smiles>

NCI0262634

3

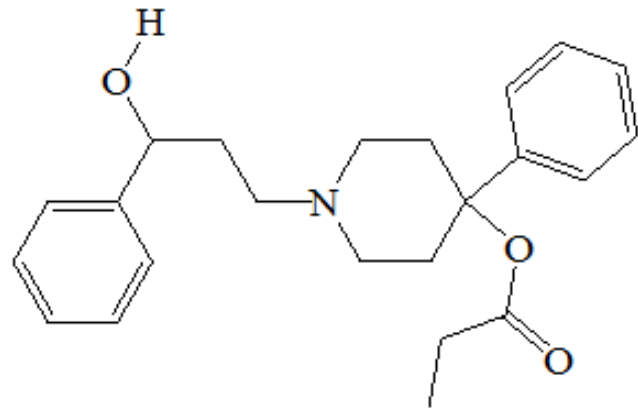

NCI0167765<smiles>CC1OC(OC2CCCc3c(O)c4c(c(O)c32)C(=O)c2ccccc2C4=O)CC(N)C1O</smiles>

NCI0221267

2<smiles>O=C1c2ccccc2C(=O)c2c3c(cc(NCCNCCO)c21)N(CCO)CCN3</smiles>

NCI0270924<smiles>CC1(C)N=C(N)N=C(N)N1c1cccc(C(=O)Nc2cccnc2)c1</smiles>

NCI0128190

Figure 3. Chemical structures of the tested highest ranking hits captured. 


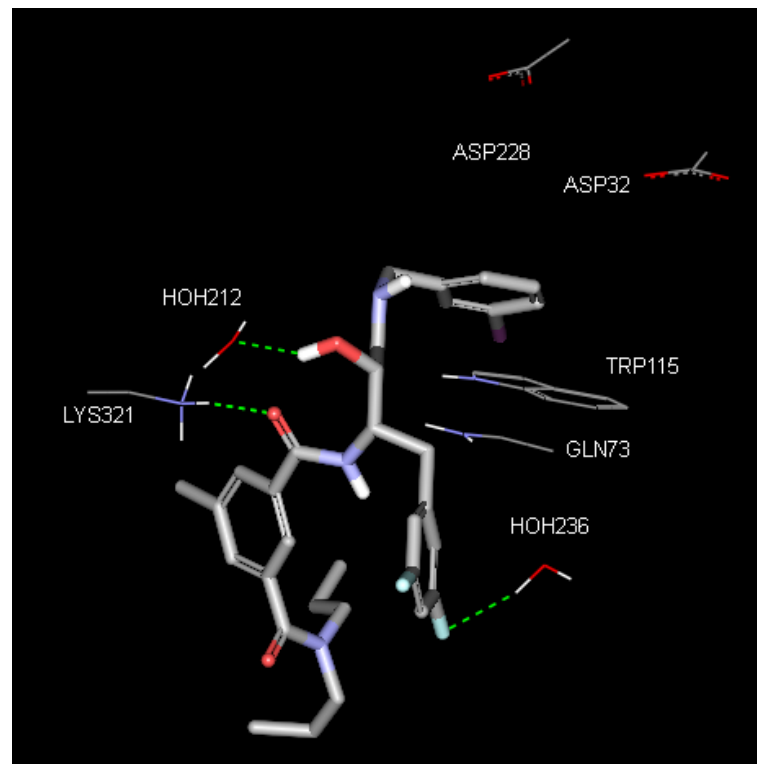

A

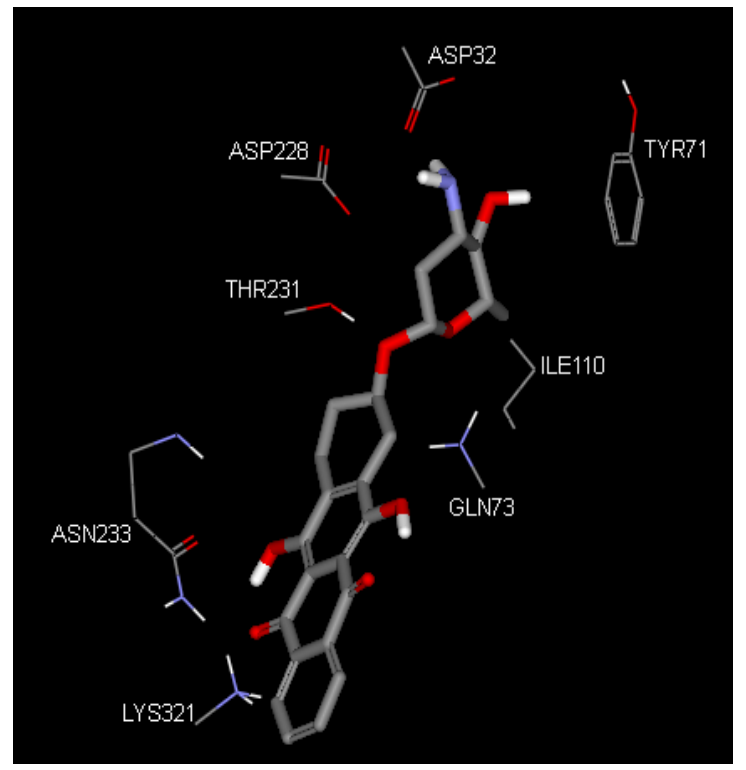

$\mathrm{B}$

Figure 4. Detailed view of: (A) compound F2I and (B) compound 3: the docked structure and the corresponding interacting amino acid moieties within the binding site of Memapsin 2IRZ.

Figure 4 shows F21 interactions as a result of docking. We can find the most important features as with IO2 ; electrostatic with ASP228 and ASP32 ; aromatic with TRP115 indole side chain ; hydrogen bond interaction connecting water molecule 212 as well as LYS321 -NH3 side chain and hydrogen bond interaction can connect water 236 then GLN73. Compound 6 display different pose inside 2IRZ this can be showed through figure 4: ionic with ASP228 and ASP32; hydrogen bond interaction connecting TYR71, THR231, GLN73, ASN233 as well as LYS321 and van der Waal interaction linking ILE110 .

Compound 6 shows the lowest binding energy value among all actives (table 1 ) with consensus score of 7 . Table 1 can tell that it is not the only parameter that can justify potency, other parameters can be considered here: F21 has higher binding energy than 6 with higher potency and consensus value of 6 . We can notice that each value for individual scoring function here can affect activity: F21 has higher scoring values for PLP1; PLP2; Jain and PMF (124.06, $123.51,10.34$, and 145.59 respectively) while compounds 6 values are: $83.62,94.17,6.82$ and 118.68 ).

PLP is simple scoring function which has been shown to correlate fine with protein-ligand binding affinities; Jain evaluated protein-ligand complexes by using an empirical scoring function and PMF is a statistical analysis approach using 3D structure databases to provide a fast and accurate prediction of protein-ligand binding free energies [34]. While LigScore1 uses the partial charges on the atoms of both ligand and receptor to determine whether an atom is polar or nonpolar besides LigScore2 types atoms of ligands and proteins based on rules that employ only formal charges, ignoring partial charges[34].

It is obvious that it is not the value of scoring function only is important, as table 1 showed that compound $\mathbf{2}$ has values that are close to F21while it is not potent, with higher binding energy. Moreover compound 6 has binding energy of $158 \mathrm{kcal} / \mathrm{mol}$ which approaches $\mathbf{F} 21$ value with $\mathrm{IC}_{50}$ of $108.9 \times 10^{2} \mu \mathrm{M}$. We may propose different binding mode as amino acids interaction are different (fig.4) and our hits does not have any interaction connecting water molecules which can be noticed with F21 or aromatic interaction observed with IO2 (fig. 3).

\section{Conclusions}

In the current project we performed a molecular docking study using LigandFit Docking and scoring as preliminary in-silico screening test for National Cancer Institute (NCI) database using binding pocket of Memapsin. The validity of this approach achieved with concomitant docking for the known Memapsin inhibitors $\mathbf{I O 2}$ and F21, followed by in-vitro enzyme assay for captured hits. As a result we recognized low micro molar inhibitor of $11 \mu \mathrm{M}$.

One of the most challenging problems in docking and scoring is the treatment of flexible receptors. Our results clearly show that Memapsin adopts different conformations depending on which ligand it binds to, which match well with literature data about Memapsin. As a consequence, docking using a rigid receptor representation corresponding to a single receptor conformation will fail for those ligands that require a different protein conformation in order to bind.

Docking is useful in decreasing a collection of virtual compounds down to a controllable number to be synthesized and in selecting compounds from an external data library. Virtual screening is important, as active compounds may be identified by one technique and not by the other even if experimental high-throughput screening is proposed. An added bonus is that virtual screening is fast and inexpensive 
by any standard. It is recommended, if at all possible, to use docking in parallel with other techniques such as pharmacophore modeling, to dock to multiple conformations of the target, and to use the docking results to select as many compounds as possible for experimental confirmation. As well as to use docking algorithm that deals with receptors as flexible targets.

\section{Acknowledgements}

This project was sponsored by the Deanship of Scientific Research at the Applied Science University research project (Grant No. DRGS-2013-2014-22). The authors wish to thank the Deanship of Scientific Research at the Applied Science University and Hamdi-Mango Center.

\section{Declaration of Interest}

The authors report no declarations of interest.

\section{Supporting Material}

\section{S.1 Molecular Modeling}

\section{S.1.1 Software and Hardware}

The following software packages were utilized in the present research.

- CS ChemDraw Ultra (Version 11.0), Cambridge Soft Corp., USA.

- DiscoveryStudio (DS 2.5), Accelrys, Inc., USA.

- Ligandfit within CERIUS2 (Version 4.10), Accelrys, Inc., USA.

- Libdock within Discovery Studio (Version 2.0), Accelrys, Inc., USA.

- Catalyst (version 5.11), Accelrys, Inc., USA.

\section{S.1.2 Preparation of Crystal Structures}

The 3-D coordinate of Memapsin was retrieved from the Protein Data Bank ( PDB code: 2IRZ, resolution:1.8 $\AA$ [50]. Hydrogen atoms were added to the protein utilizing DS 2.0 templates for protein residues. Gasteiger-Marsili charges were assigned to the protein atoms as implemented within DS $2.5[33,34]$. The protein structures were utilized in subsequent docking experiments without energy minimization. Explicit water molecules were kept, i.e., docking in the presence of explicit water molecules.

\section{S.1.3. Docking Configurations}

\section{(1). LigandFit Docking and Scoring}

LigandFit considers the flexibility of the ligand and treats the receptor as rigid. There are two steps implemented in the LigandFit process:

(A) Defining the location(s) of potential binding site(s)
$[35,36]$. In the current docking experiments, the binding site was generated from the cocrystallized ligand $\mathbf{I O 2}$ 3-\{5-[(1r)-1-Amino-1-Methyl-2-Phenylethyl]-

1,3,4-Oxadiazol-2-Y1\}-N-[(1r)-1-(4-Fluorophenyl)Ethyl]-

5-[Methyl(Methylsulfonyl) Amino] Benzamide within the targeted protein(fig. 2).

(B) Docking the ligands in the binding site [35-36].

In LigandFit, docking is composed of few substeps: (i) Conformational search of flexible ligands employing Monte Carlo randomized process. (ii) Pose and conformation selection based on shape similarity with the binding site. (iii) Candidate conformers and poses exhibiting low shape discrepancy are further enrolled in calculation of the dock energies.

(iv) Each docked conformation and pose is further fitted into the binding pocket through a number of rigid-body minimization iterations. (v) Docked conformers and poses that have docking energies below certain user-defined threshold are subsequently clustered according to their rms similarities. Representative conformers and poses are then selected, further energy-minimized within the binding site, and saved for subsequent scoring.

In the current docking experiments NCI hits in their un-ionized form was docked into the binding site with a nonbonded cutoff distance of $10.0 \AA$ and distance dependent dielectric. The interaction energy was estimated by a trilinear interpolation value using soft potential energy approximations [35].

- Rigid body ligand minimization parameters: 30 steepest descend followed by 60 BFGS minimization iterations were applied to every orientation of the docked ligand. The best 10 poses were further energy minimized within the binding site for a maximum of 300 rigid body iterations.

- High-ranking docked conformers and poses were scored using seven scoring functions: Jain [37], LigScore1, LigScore2 [35, 38], PLP1[39], PLP2 [39], PMF, and PMF04 [40-42].

LigScore1 and LigScore2 scores were calculated employing CFF force field (version 1.02) and using grid-based energies with a grid extension of $7.5 \AA$ across the binding site. PMF scores were calculated employing cutoff distances of $12.0 \AA$ for carbon-carbon interactions and other atomic interactions, while PMF04 scores were calculated employing cutoff values of 6.0 and $9.0 \AA \AA$ for carbon-carbon interactions and other atomic interactions, respectively.

(2). LibDock Docking. LibDock docks ligands (after removing hydrogen atoms) into a binding site guided by binding hotspots. It aligns docked ligand conformations to polar and apolar receptor interactions sites, i.e., hotspots. Conformations can be either pre-calculated or generated on the fly. Because some of the output poses may have hydrogen atoms in close proximity to the receptor, a CHARMm minimization step can be optionally enabled to further optimize the docked poses [43-44]. LibDock performs the following steps using a set of pre-generated 
ligand conformations and a receptor with a specified binding site: [43-44]. (i) Remove hydrogen atoms. (ii) Rank ligand conformations and prune by solvent accessible surface area (SASA). (iii) Find hotspots using a grid placed into the binding site and using polar and apolar probes. The numbers of hotspots are pruned by clustering to a user defined value. (iv) Dock ligand poses by aligning to the hotspots. This is performed by using triplets (i.e., three ligand atoms are aligned to three receptor hotspots). Poses which result in protein clashes are removed. (v) A final BFGS pose optimization stage is performed using a simple pairwise score. The top scoring ligand poses are retained. (vi) Hydrogen atoms are then added back to the docked ligands.

(vii) Optionally, CHARMm minimization can be carried out to reduce steric clashes caused by added hydrogen atoms.

In the current docking experiments we employed the following parameters.

- Binding site sphere radius of $11.8 \AA$ surrounding the center of the co-crystallized ligand (IO2) (fig. 2).

- Ligand-to-hotspots matching rmsd tolerance value was set to $0.25 \AA$.

- Maximum number of saved poses for each ligand $=$ 100.

- Maximum number of poses saved for each ligand during hotspots matching before entering the final pose minimization $=100$.

- Minimum LibDock score (poses below this score are not reported) $=100$.

- $\quad$ Fraction of reported top scoring poses $=0.5$.

- Maximum number of rigid body minimization steps during final pose optimization phase (using BFGS method) $=50$.

- Maximum number of evaluated poses for each conformation $=30$.

- Maximum number of steric clashes allowed before the pose-hotspot alignment is terminated (specified as a fraction of the heavy atom count $)=0.10$.

- Cluster similarity cutoff value $=0.5 \AA$ (docked poses are rigid-body minimized and clustered using this cutoff value).

- Maximum value for nonpolar solvent accessible surface area for a particular pose to be reported as successful $=15.0 \AA^{2}$.

- Maximum value for polar solvent accessible solvent area for a particular pose to be reported as successful $=$ $5.0 \AA^{2}$.

- Number of grid points used for calculating solvent accessible surface area $=18$.

- Conformation generation method: The CATALYST module CATCONFIRM implemented in DS 2.0 was implemented employing the BEST conformation generation option to ensure the best coverage of the compound's conformational space. Maximum number of conformations to be generated per ligand $=255$ not exceeding an energy threshold of $20 \mathrm{kcal} / \mathrm{mol}$ from the most stable conformer. No final ligand minimization was implemented (i.e., in the binding pocket).
- The docked poses were scored employing the same seven scoring functions that were implemented in LigandFit docking experiment and employing identical parameters.

\section{S.2 In Vitro Memapsin Enzyme Inhibition Assay.}

\section{S.2.1 Materials}

Materials were purchased from corresponding companies (Sigma-Aldrich and BDH Laboratory Supplies) and were used in the experimentation without further purification. Memapsin assay kit (Sigma-Aldrich CS0010) Dimethylsulfoxide (DMSO, BDH Laboratory Supplies, England). NCI hits were kindly donated by the National Cancer Institute.

\section{S.2.2 Preparation of Hit Compounds for In Vitro Assay}

The tested compounds were provided as dry powders. They were initially dissolved in DMSO to provide $0.02 \mathrm{mM}$ stock solutions and subsequently diluted to the required concentrations using $50 \mathrm{mM}$ sodium acetate, $\mathrm{pH}$ 4.5. The inhibition of Memapsin activity by the hit compounds was measured using the fluorometric assay. The final concentration of DMSO was adjusted to be less than $0.1 \%$.

\section{S.2.3 Quantification of Memapsin Activity in a Fluorometric Assay}

The Memapsin fluorescence resonance energy transfer (FRET) assay was performed as described by the manufacturer (Sigma, CS0010) (Sigma-Aldrich). Principle of the Assay: The substrate is linked to a fluorescent dye on one end and to a quenching group on its other end. The fluorescence of the substrate is significantly reduced due to intramolecular resonance energy transfer to the quenching group. Upon substrate cleavage by the enzyme, there is a disturbance of the energy transfer resulting in the enhancement of the fluorescent signal. The assay procedure can be described briefly as follows The Memapsin substrate is prepared in the buffer to a concentration of $50 \mu \mathrm{M}$. Memapsin enzyme is prepared in the same buffer to a concentration of approximately 0.3 units $/ \mu 1$. Stock solutions of test samples are prepared in DMSO, and then serially diluted in the buffer to give the desired working concentrations. Triton X-100 was added to each well to a final concentration of $160 \mathrm{M}$. Memapsin enzyme, substrate, standard, test samples and buffer are then added to the wells for a total volume of 100L, with the Memapsin enzyme being added last, just prior to reading. Baseline fluorescence is recorded immediately after the addition of the Memapsin enzyme on a fluorometer set at excitation $320 \mathrm{~nm}$, emission $405 \mathrm{~nm}$. The reaction rate was monitored for $2 \mathrm{~h}$ at $37^{\circ} \mathrm{C}$ using FLX800TBI Microplate Fluorimeter (BioTek Instruments, Winooski, USA) and the linear time-relative fluorescence units (RFU) sections were taken for rate calculation $[45,46]$.

\section{S.2.4 Memapsin Inhibition by Hit Compounds}

The inhibition of Memapsin activity by the hit compounds 
was measured using the fluorometric assay described above. The percentage of residual activity of Memapsin was determined for each compound by comparing the activity of Memapsin in the presence and absence of the tested compound. Blank and standard inhibitor (Lys-Thr-Glu-Glu-Ile-Ser-Glu-Val-Asn-Sta-Val-Ala-Glu-P he) (Sigma-Aldrich, product (A1847) was used as negative and positive controls, respectively. Measurements were conducted at least in duplicates.

\section{REFERENCES}

[1] Brookmeyer, R.; Johnson, E.; Ziegler-Graham, H.; Arrighi, H. M. (2007). Alzheimer's \&Dementia: J. Alzheimer's Assoc. 3, 186.

[2] Petersen, R.C. (2004). Mild cognitive impairment as a diagnostic entity. J Intern Med;256 (3):183-94 Sep.

[3] Luck T, Luppa M, Briel S, Riedel-Heller SG. (2010) Incidence of mild cognitive impairment: a systematic review. Dement Geriatr Cogn Disord;29(2):164-75.

[4] Ballard J. (2010) Forgetfulness and older adults: concept analysis. J Adv Nurs;66(6): 1409-19 Jun.

[5] Hardy J. and Selkoe D. J. (2002). The amyloid hypothesis of Alzheimer's disease: progress and problems on the road to therapeutics. Science 297, 353-356.

[6] Tiraboschi P.; Hansen L.A.; Thal L.J.; Corey-Bloom J. (2004). The importance of neuritic plaques and tangles to the development and evolution of AD. Neurology, 62: 1984-89.

[7] Waldemar G.; Dubois B.; Emre M.; Georges J.; McKeith I.G.; Rossor M. (2007). Recommendations for the diagnosis and management of Alzheimer's disease and other disorders associated with dementia:EFNS guideline. Eur. J. Neurol. 14: $1-26$.

[8] Bulbarelli A.; Lonati E.; Brambilla A.; Orlando A.; Cazzaniga E.; Piazza F.; Ferrarese C. ; Masserini M.; Sancini G. (2012) Aß42 production in brain capillary endothelial cells after oxygen and glucose deprivation., Molecular and Cellular Neuroscience. 49: 415-422

[9] Claeysen S., Cochet M., Donneger R., Dumuis A., Bockaert J., Giannoni P. (2012) Alzheimer culprits: Cellular crossroads and interplay Cellular Signalling 24 1831-1840

[10] Monenschein H., Horne D. B., Bartberger M. D., Hitchcock S.A., Nguyen T. T. , Patel V. F., Pennington L.D. , Zhong W. (2012) Structure guided P10 modifications of HEA derived inhibitors for the treatment of Alzheimer's disease Bioorganic \& Medicinal Chemistry Letters 22 3607-3611

[11] Xiong G.; Doraiswamy P.M. (2005). Combination drug therapy for Alzheimer's disease: what is evidence-based, and what is not? Geriatrics, 60 (6): 22-6.

[12] Zec R.F.; Burkett N.R. (2008). Non Pharmacological and Pharmacological treatment of the cognitive and behavioral symptoms of Alzheimer disease. NeuroRehab. 23: 425-38.

[13] Christopher J. Langmead, Jeannette Watson, Charlie Reavill,
(2008) Muscarinic acetylcholine receptors as CNS drug targets Pharmacology \& Therapeutics 117: 232-243

[14] Prerna U.; Vikas S. and Mushtaq A. (2010) Therapy of Alzheimer's disease: An update. African Journal of Pharmacy and Pharmacology. 4(6), 408-421.

[15] Muir, J. L. (1997). Acetylcholine, aging, and Alzheimer's disease. Pharmacol. Biochem. Behav. 56:687-696.

[16] Beach, T. G.; Honer, W. G., and Hughes L. H. (1997). Cholinergic fibre loss associated with diffuse plaques in the non-demented elderly: the preclinical stage of Alzheimer's disease? Acta. Neuropathol .93(2), 146-153.

[17] Shinoe, T.; Matsui, M.; Taketo, M. M. \& Manabe, T. (2005). Modulation of synaptic plasticity by physiological activation of M1 muscarinic acetylcholine receptors in the mouse hippocampus. J. Neurosci. 30: 11194-11200.

[18] Ibach, B., \& Haen, E. (2004). Acetylcholinesterase inhibition in Alzheimer's disease. Curr. Pharm. Des. 10(3), 231-251.

[19] Alvin V. Terry Jr., Patrick M. Callahan, Brandon Hall, Scott J. W. (2011) Alzheimer's disease and age-related memory decline [preclinical], Pharmcol. Biochem. Be. 99:190-210

[20] Wen-Hai, H.; Rong, S.; Yong-Zhou, H. (2009) Progress in the Development of Nonpeptidomimetic BACE 1 Inhibitors for Alzheimers Disease . Curr. Med. Chem. 16:1806.

[21] Cumming, J. N.; Le, T. X.; Babu, S.; Carroll, X.; Chen, L.; Favreau, P.; Gaspari, T.; Guo, D. W.; Hobbs, Y.; Huang, U.; Iserloh, M. (2008) Rational design of novel, potent piperazinone and imidazolidinone BACE1 inhibitors. Bioorg. Med. Chem. Lett. 18:3236.

[22] Yoshio, H.; Hiroko, O.; Naoko, M.; Ryoji, Y.; Abdellah, Y.; Koushi, H.; Tooru, K.; Kazuki, S.; Yoshio, H.; Shoichi, I.; Yoshiaki, K. (2008) Novel non-peptidic and small-sized BACE1 inhibitors. Bioorg. Med. Chem. Lett. $18: 1654$.

[23] Lorna, P.; Andrea, C.; Francesco, C.; Federica, B.; Manuela, B.; Francesca, M.; Maurizio, R.; Vincenza, A.; Angela, R. (2008) Multi-target-directed coumarin derivatives: hAChE and BACE1 inhibitors as potential anti-Alzheimer compounds. Bioorg. Med. Chem. Lett. $18: 423$.

[24] Yoshiari, S.; Takeshi, K.; Akinori, A.; Tetsuhiro, N.; Hachiro, S (2008) Flavonols and flavones as BACE-1 inhibitors: Structure-activity relationship in cell-free, cell-based and in silico studies reveal novel pharmacophore features. . Biochim. Biophys. Acta. 1780:819.

[25] Heuisul, P.; Kyeongsik, M.; Hyo-Shin, K.; Ki, D. K.; Dongchul, L.; Sang-Won, S.; Jae-Ung, C.; Bettina, P.; Deog-Young, C. (2008) Synthesis, SAR, and X-ray structure of human BACE-1 inhibitors with cyclic urea derivatives. Bioorg. Med. Chem. Lett. 18:2900.

[26] Derek, C. C.; Joseph, R. S.; Rajiv, C.; Rebecca, C.; John, W. E.; Kristi, Y. F.; Boyd, L. H.; Yun, H.; Steve, J.; Lee, D. J.; Guixian, J.; Peter, A. L.; Michael, S. M.; Eric, S. M.; William, J. M.; Mary-Margaret, O.; Andrea, M. O.; Albert, J. R.; Kristine, S.; JunJun, W.; Eric, W.; Jonathan, B. (2008) Acylguanidine inhibitors of $\beta$-secretase: Optimization of the pyrrole ring substituents extending into the S1 and S3 substrate binding pockets. Bioorg. Med. Chem. Lett. $18: 1063$.

[27] Huang, D.; Urs, L.; Peter, K.; Marco, C.; Alcide, B.; Amedeo, 
C. (2006) In Silico Discovery of â-Secretase Inhibitors. J. Am. Chem. Soc. $128: 5436$.

[28] Arun, K. G.; Geoffrey, B.; Cynthia, H.; Reiko, K.; Dongwoo, S.; Khaja, A.; Lin, H.; Jeffrey, A. L.; Chan, N.; Gerald, K.; Jacques, E.; Jordan, T. (2001) Structure-Based Design: Potent Inhibitors of Human Brain Memapsin 2 ( $\beta$-Secretase) . J. Med. Chem. 44 :2865.

[29] (a)-Clarke, B.; Demont, E.; Dingwall, C.; Dunsdon, R.; Faller, A.; Hawkins, J.; Hussain, I.; Macpherson, D.; Maile, G.; Matico, R.; Milner, P.; Mosley, J.; Naylor, A.; O`Brien, A.; Redshaw, S.; Riddell, D.; Rowland, P.; Soleil, V.; Smith, K.; Stanway, S.; Stemp, G.; Sweitzer, S.; Theobald, P.; Vesey, D.; Walter, D. S.; Ward, J.; Wayne, G. (2008) BACE-1 inhibitors part 2: identification of hydroxy ethylamines (HEAs) with reduced peptidic character. Bioorg. Med. Chem. Lett. $18: 1017$.

[30] (b)-Clarke, B.; Demont, E.; Dingwall, C.; Dunsdon, R.; Faller, A.; Hawkins, J.; Hussain, I.; Macpherson, D.; Maile, G.; Matico, R.; Milner, P.; Mosley, J.; Naylor, A.; O`Brien, A.; Redshaw, S.; Riddell, D.; Rowland, P.; Soleil, V.; Smith, K.; Stanway, S.; Stemp, G.; Sweitzer, S.; Theobald, P.; Vesey, D.; Walter, D. S.; Ward, J.; Wayne, G. (2008) BACE-1 inhibitors part 1: identification of novel hydroxy ethylamines (HEAs). Bioorg. Med. Chem. Lett. $18: 1011$.

[31] Beswick, P.; Charrier, N.; Clarke, B.; Demont, E.; Dingwall, C.; Dunsdon, R.; Faller, A.; Gleave, R.; Hawkins, J.; Hussain, I.; Johnson, C. N.; Macpherson, D.; Maile, G.; Matico, R.; Milner, P.; Mosley, J.; Naylor, A.; O`Brien, A.; Redshaw, S.; Riddell, D.; Rowland, P.; Skidmore, J.; Soleil, V.; Smith, K. J.; Stanway, S.; Stemp, G.; Stuart, A.; Sweitzer, S.; Theobald, P.; Vesey, D.; Walter, D. S.; Ward, J.; Wayne, G. (2008) BACE-1 inhibitors part 3: identification of hydroxy ethylamines (HEAs) with nanomolar potency in cells. Bioorg. Med. Chem. Lett. $18: 1022$.

[32] Godemann, R.; Madden, J.; Kramer, J.; Smith, M. A.; Fritz, U.; Hesterkamp, T.; Barker, J.; Hoeppner, S.; Hallett, D.; Cesura, A.; Ebneth, A.; Kemp, (2009) Fragment-Based Discovery of BACE1 Inhibitors Using Functional Assays J.Biochemistry-Us, $48: 10743$.

[33] (a)-Charrier, N.; Clarke, B.; Cutler, L.; Demont, E.; Dingwall, C.; Dunsdon, R.; Hawkins, J.; Howes, C.; Hubbard, J.; Hussain, I.; Maile, G.; Matico, R.; Mosley, J.; Naylor, A.; O`Brien, A.; Redshaw, S.; Rowland, P.; Soleil, V.; Smith, K. J.; Sweitzer, S.; Theobald, P.; Vesey, D.; Walter, D. S.; Wayne, G. (2009) Second generation of BACE-1 inhibitors Part 1: The need for improved pharmacokinetics. Bioorg. Med. Chem. Lett. 19:3664.

[34] (b)-Charrier, N.; Clarke, B.; Demont, E.; Dingwall, C.; Dunsdon, R.; Hawkins, J.; Hubbard, J.; Hussain, I.; Maile, G.; Matico, R.; Mosley, J.; Naylor, A.; O'Brien, A.; Redshaw, S.; Rowland, P.; Soleil, V.; Smith, K. J.; Sweitzer, S.; Theobald, P.; Vesey, D.; Walter, D. S.; Wayne, G. (2009) Second generation of BACE-1 inhibitors part 2: Optimisation of the non-prime side substituent. Bioorg. Med. Chem. Lett. $19: 3669$.

[35] (c)-Charrier, N.; Clarke, B.; Cutler, L.; Demont, E.; Dingwall, C.; Dunsdon, R.; Hawkins, J.; Howes, C.; Hubbard, J.; Hussain, I.; Maile, G.; Matico, R.; Mosley, J.; Naylor, A.; O‘Brien, A.; Redshaw, S.; Rowland, P.; Soleil, V.; Smith, K. J.; Sweitzer, S.; Theobald, P.; Vesey, D.; Walter, D. S.; Wayne, G. (2009) Second generation of BACE-1 inhibitors part 3: Towards non hydroxyethylamine transition state mimetics. Bioorg. Med. Chem. Lett. 19:3674.

[36] Gasteiger, J.; Marsili, M.( 1980) Iterative partial equalization of orbital electronegativity: A rapid access to atomic charges. Tetrahedron. 36:3219-3228.

[37] Discovery Studio, (2009) version 2.5; Accelrys, Inc.: San Diego.

[38] Venkatachalam, C. M.; Jiang, X.; Oldfield, T.; Waldman, M.( 2003) LigandFit: A novel method for the shape-directed rapid docking of ligands to protein active sites. J. Mol. Graphics Modell. 21:289-307.

[39] Gehlhaar, D. K.; Verkhivker, G. M.; Rejto, P. A.; Sherman, C. J.; Fogel, D. B.; Fogel, L. J.; Freer, S. T.( 1995) Molecular recognition of the inhibitor AG-1343 by HIV-1 Protease: Conformationally flexible docking by evolutionary programming. Chem. Biol. 2: 317-324.

[40] Jain, A. N.( 1996) Scoring non-covalent protein-ligand interactions: A continuous differentiable function tuned to compute binding affinities. J. Comput.-Aided Mol. Des. 10: 427-440.

[41] Krammer, A.; Kirchhoff, P. D.; Jiang, X.; Venkatachalam, C. M.;Waldman, M. (2005) LigScore: A novel scoring function for predicting binding affinities. J. Mol. Graphics Modell. 23, $395-407$.

[42] Gehlhaar, D. K.; Bouzida, D.; Rejto, P.( 1999) Reduced dimensionality in ligand-protein structure prediction: Covalent inhibitors of serine proteases and design of site-directed combinatorial libraries. In Rational Drug Design: Novel Methodology and Practical Applications; Parrill, L.; Rami Reddy, M.; Eds.; ACS Symposium Series 719; American Chemical Society; Washington, DC, 292-311

[43] Muegge, I. (2000)A knowledge-based scoring function for proteinligand interactions: Probing the reference state. Perspect. Drug Discov. 20: 99-114.

[44] Muegge, I.( 2001) Effect of ligand volume correction on PMF scoring. J. Comput. Chem., 22, 418-425.

[45] Muegge, I. (2006) PMF scoring revisited. J. Med. Chem. 49:5895-902

[46] Diller, D. J.; Merz, K. M. (2001) High throughput docking for library design and library prioritization. Proteins, 43, 113-124.

[47] Rao, S. N.; Head, M. S.; Kulkarni, A.; LaLonde, J. M. (2007) Validation studies of the site-directed docking program LibDock. J. Chem. Inf. Model. 47: 2159-2171.

[48] Al-Nadaf, A.; Abu Sheikha, G.; Taha, M.O. (2010) Elaborate ligand-based pharmacophore exploration and QSAR analysis guide the synthesis of novel pyridinium- based potent beta-secretase inhibitory leads Bioorg. Med. Chem. $18: 3088-3115$

[49] Al-Nadaf A.; Taha M. O. (2012) Ligand-based pharmacophore exploration and QSAR analysis of transition state analogues followed by in silico screening guide the discovery of new sub-micromolar Beta-Secretase inhibitors. Med. Chem. Res. 22(4):1979-1997, 2013.

[50] Lipinski C.,F.Lombardo, B.Dominy, P.Feeney, Experimental and computational approaches to estimate solubility and permeability in drug discovery and development settings, J.Adv. Drug Deliv.Rev.46(2001)3-26.

[51] Veber D., S.Johnson, H.Cheng, B.Smith, K.Ward, K.Kopple, 
(2002) Molecular proper-ties that influence the oral bioavailability of drug candidates, J.Med.Chem.45 2615-2623.

[52] Sinha S.; John P. A.; Barbour R.; Basi G. S.; Caccavello R.; Davis D.; Doan M.; Dovey H. F.; Frigon N.; Hong J.; Jacobson-Croak K.; Jewett N.; Keim P.; Knops J. ; Lieberburg I.; Power M.; Tan H.; Tatsuno G.; Tung J.; Schenk D.; Seubert P.; Suomensaari S. M.; Wang S. ; Walker D.; Zhao J.; McConlogue L. and John V.( 1999) Purification and cloning of amyloid precursor protein b-secretase from human brain. NATURE. 402:537-540
[53] Rajapakse HA, Nantermet PG, Selnick HG, Munshi S, McGaughey GB, Lindsley SR, Young MB, Lai MT, Espeseth AS, Shi XP, Colussi D, Pietrak B, Crouthamel MC, Tugusheva K, Huang Q, Xu M, Simon AJ, Kuo L, Hazuda DJ, Graham S, Vacca JP. (2006) Discovery of oxadiazoyl tertiary carbinamine inhibitors of beta-secretase (BACE-1). J Med Chem. Dec 14;49(25):7270-3.

[54] Al-Nadaf A.H., Taha M. O. and Aldal'in H. K., Haloperidol inhibits memapsin 2: Innovation by docking simulation and In vitro assay(2015), Pak. J. Pharm. Sci., 28:1, 139-146 